\title{
Computational insights into molecular mechanisms of CD95 programmed cell death activation
}

\author{
Nikita V. Ivanisenko \\ Institute of Cytology and Genetics \\ SB RAS, Novosibirsk, Russia \\ n.ivanisenko@gmail.com \\ Vladimir A. Ivanisenko \\ Institute of Cytology and Genetics \\ SB RAS, Novosibirsk, Russia
}

Laura K. Hillert

Translational Inflammation Research, Medical Faculty, Otto von Guericke

University Magdeburg, Magdeburg, 39106, Germany

Corinna König

Translational Inflammation Research, Medical Faculty, Otto von Guericke University Magdeburg, Magdeburg, 39106, Germany

\author{
Inna N. Lavrik \\ Translational Inflammation Research, \\ Medical Faculty, Otto von Guericke \\ University Magdeburg, Magdeburg, \\ 39106, Germany \\ Institute of Cytology and Genetics \\ SB RAS, Novosibirsk, Russia
}

\begin{abstract}
The assembly of the death-inducing signaling complex (DISC) and death effector domain (DED) filaments at CD95/Fas results in activation of extrinsic apoptosis. This process is deregulated in the variety of tumors and neurodegenerative diseases. In this work computational modeling was carried out to describe the molecular mechanism of DISC assembly and to develop small molecules facilitating cell death induction.
\end{abstract}

Keywords - CD95, cell death, apoptosis, c-FLIP, virtual screening, docking.

\section{Introduction}

There are two types of apoptosis induction: intrinsicmediated via mitochondria and extrinsic-mediated via death receptor (DR) activation. Currently six DRs are characterized: CD95/Fas, TNF-R1, TRAILR1/2, DR3 and DR6, while CD95/Fas is one of the most studied members of the DR family. The induction of apoptosis via CD95 is largely controlled by the Death-Inducing Signaling Complex (DISC), which is formed upon CD95 stimulation. CD95 DISC comprises oligomerized, CD95, the adaptor protein FADD, procaspases-8/10 and cellular FLICE inhibitory proteins (cFLIP). Deregulation of the CD95 pathway accompanies a variety of tumors and neurodegenerative diseases. Currently a limited number of small-molecule agents targeting this pathway is available. Molecular modeling of the components of DISC complex and development of compounds targeting them is of great interest and the goal of the current work.

\section{Methods}

Virtual screening was conducted using GLIDE software (Schrodinger Inc.). Molecular modeling was carried out using Rosetta Software.

\section{Results}

Using virtual screening techniques of large databases of chemical compounds we could identify first in class small molecule targeting heterodimer caspase-8/c-FLIPL [1]. Chemical probe was designed to target the heterodimerization interface leading to allosteric stabilization of the complex. Kinetic mathematical model was further developed to analyze the observed effects of FLIPinB $\gamma$ on DISC activation. Based on the modeling results we could predict that the stabilized FLIPinB $\gamma /$ caspase-8/c-FLIPL complex plays a major role at the very initial stages of the DED chain assembly and procaspase- 8 processing. Furthermore conducted structural analysis of the DISC complex [2-3] suggests high therapeutic potential of c-FLIP targeting compounds to enhance cell death in cancer cell lines that are characterized by high c-FLIP levels.

\section{ACKNOWLEDGMENT}

The study has been financially supported by RFBR grant (No.19-54-45015).

\section{REFERENCES}

[1] Hillert, L.K., Ivanisenko, N.V., Busse, D., Espe, J., König, C., Peltek, S.E., Kolchanov, N.A., Ivanisenko, V.A. and Lavrik, I.N., 2020. Dissecting DISC regulation via pharmacological targeting of caspase8/c-FLIP L heterodimer. Cell Death \& Differentiation, pp.1-14.

[2] Hillert, L.K., Ivanisenko, N.V., Espe, J., König, C., Ivanisenko, V.A., Kähne, T. and Lavrik, I.N., 2020. Long and short isoforms of c-FLIP act as control checkpoints of DED filament assembly. Oncogene, 39(8), pp.1756-1772.

[3] Ivanisenko, N.V., Buchbinder, J.H., Espe, J., Richter, M., Bollmann, M., Hillert, L.K., Ivanisenko, V.A. and Lavrik, I.N., 2019. Delineating the role of c-FLIP/NEMO interaction in the CD95 network via rational design of molecular probes. BMC genomics, 20(3), p.293. 